\title{
Associations of Baltic Sea and Mediterranean dietary patterns with bone mineral density in elderly women
}

\author{
Arja T Erkkilä ${ }^{1, *}$, Homa Sadeghi ${ }^{1}$, Masoud Isanejad ${ }^{1}$, Jaakko Mursu ${ }^{1}$, \\ Marjo Tuppurainen ${ }^{2}$ and Heikki Kröger ${ }^{3,4}$ \\ ${ }^{1}$ Institute of Public Health and Clinical Nutrition, University of Eastern Finland, Yliopistonrantra 1C, PO Box 1627, \\ FI70211 Kuopio, Finland: ${ }^{2}$ Department of Obstetrics and Gynaegology, Kuopio University Hospital, Kuopio, \\ Finland: ${ }^{3}$ Kuopio Musculoskeletal Research Unit, University of Eastern Finland, Kuopio, Finland: ${ }^{4}$ Department of \\ Orthopaedics and Traumatology, Kuopio University Hospital, Kuopio Finland
}

Submitted 22 December 2016: Final revision received 9 June 2017: Accepted 19 June 2017: First published online 14 August 2017

\begin{abstract}
Objective: Dietary quality in relation to bone health has been analysed in relatively few studies. The current study aimed to assess the association of the Baltic Sea diet (BSD) and the Mediterranean diet (MD) with bone mineral density (BMD) among elderly women.

Design: Lumbar, femoral and total body BMD were measured by dual-energy X-ray absorptiometry at baseline and year 3. Dietary intake was measured by $3 \mathrm{~d}$ food record at baseline. BSD and MD scores were calculated from food and alcohol consumption and nutrient intake. Information on lifestyle, diseases and medications was collected by questionnaires. Longitudinal associations of BSD and MD scores with BMD were analysed using linear mixed models.

Setting: Interventional prospective Kuopio Osteoporosis Risk Factor and Fracture Prevention study including women aged 65-71 years and residing in Kuopio province, Finland.

Subjects: Women ( $n$ 554) with mean age of 67.9 (SD 1.9) years and mean BMI of 28.8 (sD 4.7$) \mathrm{kg} / \mathrm{m}^{2}$.

Results: Higher BSD scores were associated with higher intakes of fruit and berries, vegetables, fish and low-fat dairy products, and lower intake of sausage. Higher MD scores were associated with higher consumption of fruit and berries and vegetables. BSD and MD scores were associated with higher PUFA:SFA and higher fibre intake. Femoral, lumbar or total body BMD was not significantly different among the quartiles of BSD or MD score.

Conclusion: The lack of associations suggest that Baltic Sea and Mediterranean dietary patterns may not adequately reflect dietary factors relevant to bone health.
\end{abstract}

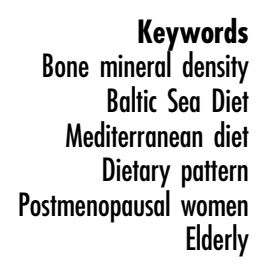

It is anticipated that in 2050 the elderly population (>60 years) will overcome the population younger than 15 years of age ${ }^{(1)}$. Osteoporosis is major public health problem, particularly in women ${ }^{(2)}$. Bone mineral density (BMD) measured by dual-energy X-ray absorptiometry is considered an important determinant of osteoporotic fractures $^{(3)}$. In Finland, the incidence of hip fracture as a major osteoporotic fracture increased dramatically until the late 1990s; afterwards, the rise levelled off until 2010. From the late 1990s to 2010, approximately 7500 hip fractures have been reported annually in Finland ${ }^{(4)}$.

Various factors such as physical activity, nutrient intake, dietary status, ethnicity, hormonal fluctuations, energy expenditure, BMI and genes may be related to BMD and risk of osteoporosis ${ }^{(5)}$. The association of diet with bone health can be analysed focusing on single nutrients such as $\mathrm{Ca}$, vitamin D or protein $^{(6,7)}$. A more holistic view on diet quality can be derived by analysing dietary patterns in relation to bone health ${ }^{(8-11)}$. Dietary patterns found through a posteriori methods using factorial or principal component analyses have been identified in many studies. Fewer studies have focused on diet quality with the aid of predefined dietary patterns such as the Mediterranean diet (MD) or the Healthy Eating Index $(\mathrm{HEI})^{(10,12,13)}$. A dietary pattern approach is justified as the impact of a single nutritional factor is usually relatively small and hard to detect; and by studying the individual nutrients, the unknown dietary factors would be disregarded and the possible interactions of nutrients may be hard to capture. Furthermore, adjustments for other nutrients may be not feasible due to their high multicollinearity. Healthy dietary patterns have shown positive relationship with BMD in some studies ${ }^{(10,12,14,15)}$, but not in all ${ }^{(13)}$. 
According to our knowledge, the relationship of the Baltic Sea diet (BSD) with BMD has not been reported earlier. This diet is recognized as a healthy eating pattern in Nordic countries (Norway, Sweden, Denmark, Finland and Iceland). The BSD is characterized mainly by high consumption of Nordic berries and fruit, whole grains, vegetables, fish, fat-free or low-fat dairy products, and lower consumption of processed meat and alcohol ${ }^{(16)}$. According to the recent studies by Kanerva et al. ${ }^{(17-19)}$ and Perälä et $a l .{ }^{(20)}$, the BSD had significant positive effects on health status. In addition to this dietary pattern relevant to Finnish food culture, we analysed adherence to the MD as a more commonly used dietary pattern. The MD is characterized by high consumption of fish, legumes, nuts, olive oil, vegetables and fruit, lower intake of full-fat dairy products and red meat, and moderate use of alcohol. The aim of the present study was to assess the association of the BSD and MD with BMD in elderly women in a followup of 3 years.

\section{Methods}

\section{Participants}

The data were from the Kuopio Osteoporosis Risk Factor and Fracture Prevention Study (OSTPRE-FPS) in Kuopio, Finland, during 2003-2007. Of the 13100 peri- and postmenopausal women born in 1932-1941, a total of 5407 were sent an invitation by mail to participate in the study. The criteria for including the participants were minimum age of 65 years at the end of November 2002, residency in Kuopio province at the beginning of the study and having not participated in earlier BMD measurements in OSTPRE. From the population, $63.5 \%$ responded to the mailed invitation and expressed interest to participate in the intervention. These 3432 women were randomized into two equal groups; one receiving $1000 \mathrm{mg} \mathrm{Ca}$ and $25 \mu \mathrm{g}$ (1000 IU) vitamin D daily and the other receiving no placebo. From these women, 750 were included randomly in a subpopulation in which BMD was measured. The results of the intervention have been published and as the intervention had a positive effect on total body $\mathrm{BMD}$, the intervention group is included in the statistical models ${ }^{(21)}$. Baseline measurements were carried out from February 2003 to May 2004, and follow-up measurements between January 2006 and May 2007. From 593 women who completed the study and their food record was attained at baseline, 544 women had BMD measurement at the femoral neck and 480 of them at the lumbar spine, the results of whom were applied in the current study.

\section{Questionnaire}

Questionnaires were posted to the participants' homes and returned at the study visit. Alcohol consumption, smoking (current, former, never smoked), current daily or almost daily use of dietary supplements, diseases, time from menopause, use of medicines such as hormone therapy and current mobility were assessed in the questionnaires. Restricted mobility was defined based on a question about current moving ability, with the options of 'fully mobile', 'able to move, but not run', 'not able to walk more than $1 \mathrm{~km}$ ', 'not able to walk more than $100 \mathrm{~m}$ ', 'able to move only indoors' and 'immobile'. To assess alcohol consumption, participants were asked to quantify their intake of beer and cider bottles ( 1 bottle equals $330 \mathrm{ml}$ ), wine glasses ( 1 glass equals $120 \mathrm{ml}$ ), spirits or strong alcohol portions of $40 \mathrm{ml}$ during the last 4 weeks. Diseases which may have affected BMD were asked in the questionnaire including hyperthyroidism, disease of the parathyroid gland, chronic liver disease, chronic intestinal disease, coeliac disease, ventricular operation, chronic nephropathy, arthritis, osteoporosis and lactose intolerance. Furthermore, medications influencing BMD, such as loop diuretics, insulin, anti-epileptics, glucocorticoids and cancer chemotherapy, were assessed using the questionnaire.

\section{Dietary assessment}

At baseline, of those participants who had BMD measurement data, 554 women produced valid dietary intake data $^{(22)}$. The participants filled in $3 \mathrm{~d}$ food records at home and returned them during the research visit. The instructions for filling the forms were sent beforehand to the participants and they were asked to record their food intake for three consecutive days, two of which were weekdays and one a weekend day. In the case of ambiguity, participants were telephoned by a nutritionist. Participants were asked for the type of fat used on bread, in cooking and in baking in separate questions. Calculation of nutrient intake was done with the software Nutrica program version 2.5 (Finnish Social Insurance Institute, Turku, Finland).

\section{Dietary scores}

BSD scoring was based on an earlier study ${ }^{(16)}$ with slight modifications due to the different dietary assessment $\operatorname{method}^{(23,24)}$. The Baltic Sea Diet Pyramid and Nordic Nutrition Recommendations were the basis for the original BSD scoring ${ }^{(16)}$. To create a BSD score, the intakes of total fruit and berries, vegetables (potatoes excluded as a starchy vegetable), fibre from total cereal products, fish, fat-free and low-fat ( $<2 \%$ fat) liquid dairy products (milk and fermented milk), sausage, ratio of PUFA to SFA, and total fat intake (percentage of energy) were categorized into quartiles. For fruit and berries, vegetables (potatoes excluded), fibre from cereal products, fish, fat-free and low-fat dairy products and PUFA:SFA, the highest points (3) were allocated to the highest quartile of consumption and 0 points to the lowest quartile; whereas for sausage and total fat the scoring was opposite, awarding highest 
points to the lowest quartile. Moreover, for alcohol consumption measured with the questionnaire, 1 or $<1$ portion/week (1 portion equals $12 \mathrm{~g}$ ) got the highest points (1), and $>1$ portion/week got the lowest points (0). Quartile scores for foods and nutrients were summed up and ranged from 0 to 25 , with higher scores indicating higher adherence to the BSD. The total score was categorized into quartiles.

MD score was defined based on the existing scores in the literature and particularly those studies that have applied the MD score in Nordic cohorts ${ }^{(25-27)}$. It was based on consumption of vegetables (potatoes excluded), fruit, cereals and potatoes, fish, ratio of PUFA plus MUFA to SFA, total meat and eggs, total milk and dairy products and alcohol (derived from the questionnaire). Consumption equalling or exceeding the median consumption was scored 1 point and lower than the median 0 points for the following food groups: vegetables, fruit, cereals and potatoes, fish and PUFA + MUFA:SFA. Scoring was opposite for meat and eggs and dairy products: consumption lower than the median consumption was scored as 1 point. Alcohol consumption of $5-25 \mathrm{~g} / \mathrm{d}$ was scored 1 point and consumption lower or higher than that scored 0 points. Scores were summed up and ranged from 0 to 8 , with higher scores indicating higher adherence to the MD. As for BSD, the total MD score was categorized into quartiles.

\section{Bone mineral density measurement}

Measurement of BMD was performed by dual-energy X-ray absorptiometry (Lunar Prodigy instrument; GE Healthcare, Madison, WI, USA) at the lumbar spine (L2-L4), femoral neck and total body ${ }^{(21)}$. Measurements were done at baseline and after 3 years by trained nurses. The quality and technical monitoring was done every day. The long-term reproducibility (CV) of the instrument for BMD during the study period, as determined by regular phantom measurements, was $0.4 \%$. Measurement errors were excluded from the analysis. Osteoporosis was defined based on WHO criteria as femoral neck T-score lower than or equal to $-2 \cdot 5$ sD of a young reference population.

\section{Antbropometric measurements}

Weight and height measurements were done at baseline by use of a calibrated scale (Philips, type HF 351700 ) and a wall meter, respectively. BMI $\left(\mathrm{kg} / \mathrm{m}^{2}\right)$ calculation was done by dividing weight (in kilograms) by the square of height (in metres).

\section{Statistical analysis}

Analysis of the data was done using the statistical software package IBM SPSS Statistics version 21. Baseline characteristics and dietary intake among the BSD and MD score quartiles were compared by one-way ANOVA and respective non-parametric test (Kruskal-Wallis test) followed by appropriate post hoc tests (Tukey), and for categorical variables by the $\chi^{2}$ test.

The associations of BSD and MD score quartiles with lumbar BMD, femoral neck BMD and total body BMD at baseline and at year 3 were analysed with the mixed model for repeated measurements. Mixed models simultaneously analyse a combination of several factors and covariates in repeated time points, and the effect of missing data is decreased and heterogeneity across individuals is taken into account ${ }^{(28)}$. We entered BMD data from baseline and year 3, as well as dietary scores and confounding factors from baseline, in the mixed model. We analysed the data using an unadjusted model and an adjusted model including smoking, intervention group, habitual vitamin $\mathrm{D}$ and $\mathrm{Ca}$ supplementation, disease or medication reducing BMD, age, weight, height, duration of hormone therapy and energy intake as potential covariates as fixed effects. Subject effect was entered as a random effect in the models. Similarly, associations of the categories of dietary score components with BMD were analysed using adjusted mixed models.

Given that about half of the women received $\mathrm{Ca}$ and vitamin D supplementation, we tested the interaction terms between BSD and MD scores with vitamin D and Ca intervention. There was no significant interaction; therefore, data were pooled for the total population adjusting for the intervention. In addition, the analyses were run only in the control group.

\section{Results}

The elderly postmenopausal women had a mean age of 67.9 (SD 1.9) years and mean BMI was 28.8 (SD 4.7) $\mathrm{kg} / \mathrm{m}^{2}$, which was in overweight range $\left(\mathrm{BMI} \geq 25 \cdot 0 \mathrm{~g} / \mathrm{m}^{2}\right)$. At baseline 123 women (22.2\%) used hormone therapy, the mean duration of hormone therapy use was 11 years, and time from menopause was 18 years. Among the women, 26 and $23 \%$ had taken self-care Ca and vitamin D supplements, respectively. There were more current smokers in the lowest BSD score quartile than in the other quartiles; however, MD quartiles were not associated with smoking (Table 1). The mean lumbar, femoral neck and total BMD were $1.096,0.869$ and $1.077 \mathrm{~g} / \mathrm{cm}^{2}$, respectively. Baseline BMD values were not significantly different across BSD or MD quartiles (Table 1). Further, there were no significant associations of BSD and MD quartiles with osteoporosis.

Table 2 describes the mean intakes of main food groups and nutrients according to the BSD and MD score quartile groups. The highest amounts of fruit and berries, vegetables, fish, fat-free and low-fat dairy products, and the lowest amount of sausage, were consumed in the highest BSD score quartile. The consumption of alcohol did not differ significantly between the BSD score quartiles. The highest BSD score was associated with the highest intakes of energy, PUFA:SFA, protein $(\mathrm{g} / \mathrm{d})$, carbohydrate 
Table 1 Baseline characteristics of participants in quartiles of Baltic Sea diet (BDS) and Mediterranean diet (MD) scores; Finnish women aged 65-71 years ( $n$ 554), Kuopio Osteoporosis Risk Factor and Fracture Prevention Study (OSTPRE-FPS), 2003-2007

\begin{tabular}{|c|c|c|c|c|c|c|c|c|c|c|c|c|c|c|c|c|c|c|}
\hline & \multicolumn{8}{|c|}{ Quartile of BSD score } & \multirow[b]{3}{*}{$P$ value* } & \multicolumn{9}{|c|}{ Quartile of MD score } \\
\hline & \multicolumn{2}{|c|}{$\begin{array}{c}\text { Q1 ( } \leq 9 \text { points }) \\
\quad(n 146)\end{array}$} & \multicolumn{2}{|c|}{$\begin{array}{c}\text { Q2 (10-13 } \\
\text { points) } \\
(n 125)\end{array}$} & \multicolumn{2}{|c|}{$\begin{array}{c}\text { Q3 (14-15 } \\
\text { points) } \\
(n 129)\end{array}$} & \multicolumn{2}{|c|}{ Q4 ( $\begin{array}{c}(\geq 16 \text { points }) \\
(n 107)\end{array}$} & & \multicolumn{2}{|c|}{$\begin{array}{c}\mathrm{Q} 1 \begin{array}{l}\text { ( } \\
(n \text { 3 points })\end{array} \\
\quad 160)\end{array}$} & \multicolumn{2}{|c|}{$\begin{array}{l}\text { Q2 (4 points) } \\
\quad(n 147)\end{array}$} & \multicolumn{2}{|c|}{$\begin{array}{c}\text { Q3 (5-6 points) } \\
\text { ( } n \text { 117) }\end{array}$} & \multicolumn{2}{|c|}{ Q4 $\underset{(\geq 7 \text { points })}{(n \text { 79) }}$} & \multirow[b]{2}{*}{$P$ value* } \\
\hline & Mean & SD & Mean & SD & Mean & SD & Mean & SD & & Mean & SD & Mean & SD & Mean & SD & Mean & SD & \\
\hline Age (years) & 67.9 & 1.9 & 67.9 & 1.8 & $68 \cdot 0$ & 1.9 & 67.5 & 1.8 & 0.145 & $67 \cdot 8$ & 1.9 & $67 \cdot 8$ & 1.8 & 67.9 & 1.9 & 68.0 & $2 \cdot 0$ & 0.915 \\
\hline Height $(\mathrm{cm})$ & $158 \cdot 1$ & $6 \cdot 0$ & $158 \cdot 1$ & $5 \cdot 1$ & 158.9 & $5 \cdot 1$ & 159.9 & 4.9 & 0.020 & $158 \cdot 1$ & $5 \cdot 0$ & 158.6 & $5 \cdot 8$ & 159.5 & $5 \cdot 2$ & $159 \cdot 4$ & $5 \cdot 1$ & 0.113 \\
\hline Weight (kg) & $72 \cdot 7$ & $12 \cdot 8$ & $70 \cdot 8$ & $10 \cdot 3$ & 73.2 & $12 \cdot 4$ & 71.7 & 11.6 & 0.375 & $72 \cdot 1$ & 11.6 & $73 \cdot 7$ & $12 \cdot 3$ & $72 \cdot 1$ & 11.5 & $70 \cdot 7$ & $12 \cdot 6$ & 0.319 \\
\hline BMI $\left(\mathrm{kg} / \mathrm{m}^{2}\right)$ & $29 \cdot 1$ & $5 \cdot 1$ & 28.4 & 4.2 & $29 \cdot 0$ & 4.6 & $28 \cdot 0$ & $4 \cdot 3$ & 0.206 & $27 \cdot 4$ & $4 \cdot 1$ & $28 \cdot 1$ & 4.5 & $27 \cdot 1$ & $4 \cdot 1$ & $26 \cdot 9$ & 4.3 & 0.163 \\
\hline $\begin{array}{l}\text { Duration of HT use (years; } \\
\quad n 266 \text { ) }\end{array}$ & $10 \cdot 3$ & 6.5 & $10 \cdot 5$ & $5 \cdot 1$ & 11.5 & $6 \cdot 4$ & $11 \cdot 0$ & $6 \cdot 0$ & 0.749 & $10 \cdot 7$ & $5 \cdot 6$ & 11.0 & $5 \cdot 8$ & $12 \cdot 1$ & $6 \cdot 6$ & $10 \cdot 5$ & $5 \cdot 7$ & 0.519 \\
\hline $\begin{array}{l}\text { Time since menopause } \\
\text { (years) }\end{array}$ & $18 \cdot 9$ & $5 \cdot 2$ & $18 \cdot 5$ & $5 \cdot 3$ & $18 \cdot 1$ & 4.9 & $18 \cdot 1$ & $5 \cdot 4$ & 0.724 & $18 \cdot 5$ & $5 \cdot 3$ & $17 \cdot 8$ & $5 \cdot 0$ & $19 \cdot 3$ & 4.9 & $18 \cdot 4$ & $5 \cdot 6$ & 0.230 \\
\hline Lumbar BMD (g/cm²) & $1 \cdot 107$ & 0.193 & 1.080 & 0.192 & 1.091 & 0.177 & $1 \cdot 108$ & 0.194 & 0.680 & 1.074 & 0.187 & $1 \cdot 130$ & 0.192 & 1.089 & 0.186 & 1.085 & 0.182 & 0.110 \\
\hline $\begin{array}{l}\text { Femoral neck BMD } \\
\left(\mathrm{g} / \mathrm{cm}^{2}\right)\end{array}$ & 0.868 & 0.138 & 0.860 & 0.120 & 0.881 & 0.124 & 0.868 & 0.123 & 0.652 & 0.866 & 0.127 & 0.886 & 0.132 & 0.864 & 0.116 & 0.856 & 0.125 & 0.290 \\
\hline Total body BMD $\left(\mathrm{g} / \mathrm{cm}^{2}\right)$ & 1.074 & 0.091 & 1.070 & 0.098 & 1.088 & 0.094 & 1.076 & 0.093 & 0.585 & 1.066 & 0.096 & 1.092 & 0.089 & 1.073 & 0.091 & 1.080 & 0.985 & 0.216 \\
\hline Osteoporosis (\%)† & \multirow{2}{*}{\multicolumn{2}{|c|}{$\begin{array}{r}3.5 \\
11.2\end{array}$}} & \multirow{2}{*}{\multicolumn{2}{|c|}{1.6}} & \multicolumn{2}{|c|}{2.4} & \multicolumn{2}{|c|}{0.0} & 0.267 & \multirow{2}{*}{\multicolumn{2}{|c|}{1.9}} & \multicolumn{2}{|c|}{3.5} & \multicolumn{2}{|c|}{0.0} & \multicolumn{2}{|c|}{$2 \cdot 6$} & 0.264 \\
\hline Current mobility limited (\%) & & & & & \multirow{2}{*}{\multicolumn{2}{|c|}{$\begin{array}{r}0.0 \\
41 \cdot 1\end{array}$}} & \multirow{2}{*}{\multicolumn{2}{|c|}{$\begin{array}{r}3.8 \\
34.0\end{array}$}} & 0.112 & \multirow{2}{*}{\multicolumn{2}{|c|}{$\begin{array}{l}10 \cdot 1 \\
35 \cdot 6\end{array}$}} & \multirow{2}{*}{\multicolumn{2}{|c|}{$\begin{array}{r}5 \cdot 6 \\
36 \cdot 1\end{array}$}} & \multirow{2}{*}{\multicolumn{2}{|c|}{$\begin{array}{r}4.3 \\
37.9\end{array}$}} & 7. & 7 & 0.257 \\
\hline $\begin{array}{l}\text { Disease or medication } \\
\text { decreasing BMD (\%) }\end{array}$ & \multicolumn{2}{|c|}{$32 \cdot 9$} & \multicolumn{2}{|c|}{$\begin{array}{r}5.0 \\
39.2\end{array}$} & & & & & 0.447 & & & & & & & \multicolumn{2}{|c|}{40.5} & 0.886 \\
\hline Ca supplementation (\%) & \multicolumn{2}{|c|}{$19 \cdot 9$} & $30 \cdot 6$ & & $23 \cdot 2$ & & $29 \cdot 6$ & & 0.157 & 25 & & 22.4 & & 28.2 & & 25 & 3 & 0.765 \\
\hline $\begin{array}{l}\text { Vitamin D } \\
\text { supplementation (\%) }\end{array}$ & 19.8 & & $27 \cdot 2$ & & $23 \cdot 3$ & & 27 & & 0.431 & 24 & & 25 & & $22 \cdot 2$ & & 19. & & 0.724 \\
\hline Smoking status & & & & & & & & & 0.021 & & & & & & & & & 0.290 \\
\hline Never (\%) & 81.7 & & 75.4 & & 85.9 & & $86 \cdot 7$ & & & $82 \cdot 6$ & & $85 \cdot($ & & $77 . \varsigma$ & & $87 \cdot$ & & \\
\hline Previous smoker (\%) & 9.8 & 9 & $20 \cdot 5$ & & $10 \cdot 9$ & & 11.4 & 4 & & $12 \cdot$ & & $8 \cdot \varepsilon$ & 8 & $17 \cdot 7$ & & 11.5 & 5 & \\
\hline Current smoker (\%) & 8.5 & 5 & 4.1 & & 3.1 & 1 & 1.5 & 9 & & 4.5 & & 6. & $\cdot 1$ & 4.4 & & 1.3 & 3 & \\
\hline
\end{tabular}

$H T$, hormone therapy; BMD, bone mineral density.

*One-way ANOVA for continuous variables and Pearson's $x^{2}$ test for categorical variables.

†T-score lower than or equal to $-2.5 \mathrm{SD}$. 


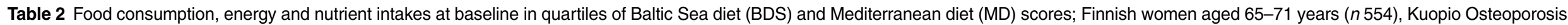
Risk Factor and Fracture Prevention Study (OSTPRE-FPS), 2003-2007

\begin{tabular}{|c|c|c|c|c|c|c|c|c|c|c|c|c|c|c|c|c|c|c|}
\hline & \multicolumn{8}{|c|}{ Quartile of BSD score } & \multirow[b]{3}{*}{$P$ value* } & \multicolumn{9}{|c|}{ Quartile of MD score } \\
\hline & \multicolumn{2}{|c|}{ Q1 ( $n$ 146) } & \multicolumn{2}{|c|}{ Q2 ( $n$ 125) } & \multicolumn{2}{|c|}{ Q3 (n 129) } & \multicolumn{2}{|c|}{ Q4 (n 107) } & & \multicolumn{2}{|c|}{ Q1 ( $n$ 160) } & \multicolumn{2}{|c|}{ Q2 (n 147) } & \multicolumn{2}{|c|}{ Q3 $(n 117)$} & \multicolumn{2}{|c|}{ Q4 ( $n$ 79) } & \multirow[b]{2}{*}{$P$ value } \\
\hline & Mean & SD & Mean & SD & Mean & SD & Mean & SD & & Mean & SD & Mean & SD & Mean & SD & Mean & SD & \\
\hline \multicolumn{19}{|l|}{ Food groups } \\
\hline Fruit and berries $(\mathrm{g} / \mathrm{d})$ & $117^{\mathrm{a}}$ & 95 & $167^{b}$ & 113 & $197^{b}$ & 120 & $252^{c}$ & 132 & $<0.001$ & $127^{\mathrm{a}}$ & 92 & $171^{\mathrm{b}}$ & 123 & $208^{\mathrm{c}}$ & 120 & $248^{\mathrm{C}}$ & 127 & $<0.001$ \\
\hline Vegetables $(\mathrm{g} / \mathrm{d})$ & $184^{\mathrm{a}}$ & 82 & $211^{\mathrm{b}}$ & 82 & $239^{b}$ & 94 & $284^{\mathrm{d}}$ & 96 & $<0.001$ & $185^{a}$ & 77 & $226^{\mathrm{b}}$ & 93 & $252^{c}$ & 88 & $276^{c}$ & 101 & $<0.001$ \\
\hline Root vegetables (g/d) & $23^{\mathrm{a}}$ & 27 & $27^{\mathrm{a}}$ & 26 & $31^{a, b}$ & 30 & $47^{b}$ & 47 & $<0.001$ & $25^{\mathrm{a}}$ & 27 & $35^{\mathrm{a}, \mathrm{b}}$ & 41 & $33^{\mathrm{a}, \mathrm{b}}$ & 33 & $38^{\mathrm{b}}$ & 31 & 0.004 \\
\hline Wholegrain bread $(\mathrm{g} / \mathrm{d})$ & 110 & 58 & 113 & 51 & 110 & 51 & 125 & 51 & 0.080 & 107 & 53 & 120 & 59 & 119 & 51 & 121 & 50 & 0.087 \\
\hline Sausage $(\mathrm{g} / \mathrm{d})$ & $29^{a}$ & 31 & $17^{\mathrm{b}}$ & 22 & $11^{\mathrm{c}}$ & 22 & $5^{c}$ & 12 & $<0.001$ & 16 & 23 & 19 & 29 & 15 & 22 & 15 & 28 & 0.525 \\
\hline Fish $(\mathrm{g} / \mathrm{d})$ & $21^{a}$ & 28 & $39^{b}$ & 37 & $47^{\mathrm{b}}$ & 42 & $67^{c}$ & 54 & $<0.001$ & $47^{\mathrm{a}}$ & 42 & $44^{\mathrm{a}}$ & 44 & $42^{a, b}$ & 47 & $29^{b}$ & 41 & 0.001 \\
\hline Low-fat dairy products $(\mathrm{g} / \mathrm{d})$ & $533^{\mathrm{a}}$ & 532 & $696^{a, b}$ & 610 & $894^{\mathrm{b}}$ & 677 & $1190^{\mathrm{c}}$ & 650 & $<0.001$ & 714 & 619 & 845 & 732 & 813 & 642 & 949 & 718 & 0.122 \\
\hline Sugar and sweets $(\mathrm{g} / \mathrm{d})$ & 21 & 14 & 22 & 16 & 19 & 15 & 19 & 13 & 0.185 & 22 & 18 & 20 & 13 & 20 & 15 & 20 & 12 & 0.880 \\
\hline Alcohol (portions/week) & 0.98 & 1.69 & 0.90 & 1.47 & 0.84 & 1.27 & 0.60 & 1.05 & 0.285 & 0.98 & 1.78 & 0.89 & 1.42 & 0.72 & 1.09 & 0.64 & 0.72 & 0.366 \\
\hline \multicolumn{19}{|l|}{ Nutrients } \\
\hline Energy (kJ/d) & $6415^{\mathrm{a}}$ & 1636 & $6448^{\mathrm{a}}$ & 1521 & $6367^{\mathrm{a}}$ & 1450 & $6952^{\mathrm{b}}$ & 1514 & 0.014 & $6272^{\mathrm{a}}$ & 1588 & $6512^{\mathrm{a}}$ & 1507 & $6714^{a, b}$ & 1542 & $7128^{\mathrm{b}}$ & 1568 & 0.001 \\
\hline Fat $(\mathrm{g} / \mathrm{d})$ & $58.7^{\mathrm{a}}$ & 19.4 & $54 \cdot 9^{\mathrm{a}, \mathrm{b}}$ & 18.0 & $50 \cdot 0^{\mathrm{b}}$ & $16 \cdot 8$ & $49.9^{\mathrm{b}}$ & $15 \cdot 6$ & $<0.001$ & 52.9 & $19 \cdot 6$ & 53.3 & 17.5 & 55.5 & 18.5 & 56.7 & $18 \cdot 3$ & 0.380 \\
\hline Fat (\% energy) & $34.7^{\mathrm{a}}$ & 4.6 & $31 \cdot 8^{\mathrm{b}}$ & 4.9 & $29 \cdot 3^{c}$ & 5.5 & $27.1^{\mathrm{d}}$ & 4.4 & $<0.001$ & 31.8 & 5.5 & 30.9 & 5.4 & 30.9 & $6 \cdot 2$ & 29.9 & 5.3 & 0.113 \\
\hline PUFA:SFA & $0.35^{\mathrm{a}}$ & 0.12 & $0.43^{\mathrm{b}}$ & 0.18 & $0.47^{c}$ & 0.14 & $0.60^{d}$ & 0.20 & $<0.001$ & $0.39^{\mathrm{a}}$ & 0.17 & $0.45^{\mathrm{b}}$ & 0.17 & $0.49^{\mathrm{b}}$ & 0.21 & $0.52^{\mathrm{b}}$ & $0 \cdot 16$ & $<0.001$ \\
\hline Protein $(\mathrm{g} / \mathrm{d})$ & $61 \cdot 2^{\mathrm{a}}$ & $16 \cdot 6$ & $65 \cdot 0^{\mathrm{a}, \mathrm{b}}$ & $16 \cdot 1$ & $69 \cdot 7^{b}$ & $16 \cdot 6$ & $78 \cdot 7^{\mathrm{c}}$ & $18 \cdot 1$ & $<0.001$ & $65 \cdot 7^{\mathrm{a}}$ & $17 \cdot 6$ & $69 \cdot 1^{a, b}$ & 18.8 & $68 \cdot 8^{\mathrm{a}, \mathrm{b}}$ & $16 \cdot 8$ & $72 \cdot 8^{\mathrm{b}}$ & 19.0 & 0.034 \\
\hline Protein (\% energy) & $16 \cdot 2^{\mathrm{a}}$ & 2.8 & $17 \cdot 2^{\mathrm{b}}$ & 2.6 & $18 \cdot 6^{\mathrm{c}}$ & $3 \cdot 1$ & $19 \cdot 3^{\mathrm{c}}$ & 3.1 & $<0.001$ & $17 \cdot 8$ & 3.4 & 18.0 & 3.2 & 17.5 & 3.2 & $17 \cdot 2$ & $2 \cdot 3$ & 0.280 \\
\hline Carbohydrate $(\mathrm{g} / \mathrm{d})$ & $181 \cdot 3^{\mathrm{a}}$ & 47.8 & $189.0^{\mathrm{a}}$ & 45.7 & $189 \cdot 8^{\mathrm{a}}$ & $46 \cdot 3$ & $216 \cdot 6^{b}$ & 48.5 & $<0.001$ & $181 \cdot 3^{\mathrm{a}}$ & $47 \cdot 1$ & $191 \cdot 3^{\mathrm{a}, \mathrm{b}}$ & $45 \cdot 2$ & $199 \cdot 2^{b}$ & 49.2 & $218 \cdot 8^{c}$ & 47.7 & $<0.001$ \\
\hline Carbohydrate (\% energy) & $46 \cdot 8^{\mathrm{a}}$ & 5.3 & $48 \cdot 8^{\mathrm{b}}$ & 5.5 & $49.7^{\mathrm{b}}$ & 6.3 & $51 \cdot 7^{\mathrm{c}}$ & 5.0 & $<0.001$ & $47.8^{\mathrm{a}}$ & 6.0 & $48 \cdot 9^{\mathrm{a}}$ & 5.4 & $49.5^{a}$ & 5.9 & $51.3^{b}$ & $5 \cdot 2$ & $<0.001$ \\
\hline Fibre $(g / d)$ & $19 \cdot 4^{\mathrm{a}}$ & 6.6 & $21 \cdot 1^{\mathrm{a}, \mathrm{b}}$ & 6.0 & $22 \cdot 5^{\mathrm{b}}$ & $6 \cdot 1$ & $26 \cdot 6^{\mathrm{C}}$ & 6.0 & $<0.001$ & $19 \cdot 5^{\mathrm{a}}$ & $6 \cdot 1$ & $22 \cdot 1^{\mathrm{b}}$ & $6 \cdot 8$ & $23 \cdot 8^{b}$ & 5.9 & $26 \cdot 2^{\mathrm{c}}$ & $6 \cdot 2$ & $<0.001$ \\
\hline $\mathrm{Ca}(\mathrm{mg} / \mathrm{d})$ & $889^{a}$ & 336 & $959^{a, b}$ & 351 & $1035^{\mathrm{b}}$ & 355 & $1183^{\mathrm{C}}$ & 383 & $<0.001$ & $957^{a}$ & 352 & $1019^{a, b}$ & 369 & $1008^{a, b}$ & 358 & $1123^{b}$ & 407 & 0.013 \\
\hline Vitamin D $(\mu \mathrm{g} / \mathrm{d})$ & $5 \cdot 3^{a}$ & 3.0 & $7 \cdot 2^{b}$ & 4.1 & $8 \cdot 1^{\mathrm{b}}$ & 4.5 & $10.5^{\mathrm{c}}$ & 6.5 & $<0.001$ & 7.8 & 4.5 & 7.8 & $5 \cdot 1$ & 7.8 & 5.4 & 7.1 & 4.6 & 0.726 \\
\hline Vitamin C $(\mathrm{mg} / \mathrm{d})$ & $72 \cdot 9^{\mathrm{a}}$ & 41.6 & $93 \cdot 6^{\mathrm{b}}$ & 52.2 & $106 \cdot 3^{\mathrm{c}}$ & 57.3 & $134.8^{\mathrm{d}}$ & 77.1 & $<0.001$ & $87.1^{\mathrm{a}}$ & 58.4 & $92 \cdot 9^{a}$ & 55.5 & $112 \cdot 1^{\mathrm{b}}$ & 70.0 & $121.0^{\mathrm{b}}$ & 54.7 & $<0.001$ \\
\hline
\end{tabular}

a,b,c,d Mean values within a row with unlike superscript letters were significantly different $(P<0.05)$.

*One-way ANOVA with Tukey's test for nutrients and Kruskal-Wallis with post hoc test for food groups, alcohol and PUFA:SFA. 
Table 3 Bone mineral density (BMD) in quartiles of Baltic Sea diet (BDS) and Mediterranean diet (MD) scores; Finnish women aged 65-71 years ( $n$ 554), Kuopio Osteoporosis Risk Factor and Fracture Prevention Study (OSTPRE-FPS), 2003-2007

\begin{tabular}{|c|c|c|c|c|c|c|c|c|c|}
\hline & \multicolumn{2}{|r|}{ Q1 } & \multicolumn{2}{|r|}{ Q2 } & \multicolumn{2}{|r|}{ Q3 } & \multicolumn{2}{|r|}{ Q4 } & \multirow[b]{2}{*}{$P$ value } \\
\hline & Mean & $95 \% \mathrm{Cl}$ & Mean & $95 \% \mathrm{Cl}$ & Mean & $95 \% \mathrm{Cl}$ & Mean & $95 \% \mathrm{Cl}$ & \\
\hline \multicolumn{10}{|c|}{ Quartile of BDS score } \\
\hline \multicolumn{10}{|c|}{ Femoral neck BMD $\left(\mathrm{g} / \mathrm{cm}^{2}\right)$} \\
\hline Unadjusted & 0.860 & $0.839,0.880$ & 0.855 & $0.833,0.878$ & 0.876 & $0.853,0.898$ & 0.862 & $0.838,0.887$ & 0.613 \\
\hline Adjusted & 0.874 & $0.846,0.901$ & 0.866 & $0.837,0.895$ & 0.892 & $0.862,0.922$ & 0.873 & $0.842,0.904$ & 0.428 \\
\hline \multicolumn{10}{|c|}{ Lumbar BMD $\left(\mathrm{g} / \mathrm{cm}^{2}\right)$} \\
\hline Unadjusted & 1.115 & $1.081,1.148$ & 1.086 & $1.049,1.122$ & 1.097 & $1.061,1.134$ & $1 \cdot 118$ & $1.078,1.158$ & 0.575 \\
\hline Adjusted & 1.136 & $1.093,1.179$ & $1 \cdot 102$ & $1.057,1.146$ & $1 \cdot 112$ & $1.065,1.159$ & $1 \cdot 135$ & $1.087,1.184$ & 0.446 \\
\hline \multicolumn{10}{|c|}{ Total body BMD $\left(\mathrm{g} / \mathrm{cm}^{2}\right)$} \\
\hline Unadjusted & 1.079 & $1.062,1.097$ & 1.072 & $1.053,1.091$ & 1.094 & $1.075,1.113$ & 1.091 & $1.072,1.111$ & 0.316 \\
\hline Adjusted & 1.078 & $1.056,1.101$ & 1.073 & $1.050,1.095$ & 1.094 & $1.071,1 \cdot 118$ & 1.088 & $1.064,1.112$ & 0.294 \\
\hline \multicolumn{10}{|c|}{ Quartile of MD score } \\
\hline \multicolumn{10}{|c|}{ Femoral neck BMD $\left(\mathrm{g} / \mathrm{cm}^{2}\right)$} \\
\hline Unadjusted & 0.859 & $0.840,0.879$ & 0.881 & $0.861,0.902$ & 0.857 & $0.834,0.881$ & 0.848 & $0.820,0.876$ & $0 \cdot 215$ \\
\hline Adjusted & 0.872 & $0.844,0.901$ & 0.883 & $0.854,0.912$ & 0.875 & $0.845,0.905$ & 0.863 & $0.827,0.898$ & 0.691 \\
\hline \multicolumn{10}{|c|}{ Lumbar BMD $\left(\mathrm{g} / \mathrm{cm}^{2}\right)$} \\
\hline Unadjusted & 1.086 & $1 \cdot 055,1 \cdot 118$ & $1 \cdot 136$ & $1 \cdot 102,1 \cdot 170$ & 1.093 & $1.055,1.130$ & 1.088 & $1.041,1.135$ & 0.143 \\
\hline Adjusted & $1 \cdot 103$ & $1.059,1.147$ & $1 \cdot 138$ & $1.093,1.182$ & $1 \cdot 121$ & $1.073,1.168$ & 1.099 & $1.042,1.157$ & 0.382 \\
\hline \multicolumn{10}{|c|}{ Total body BMD $\left(\mathrm{g} / \mathrm{cm}^{2}\right)$} \\
\hline Unadjusted & 1.072 & $1.056,1.089$ & $1 \cdot 101$ & $1.084,1 \cdot 118$ & 1.082 & $1 \cdot 062,1 \cdot 101$ & 1.078 & $1.055,1.101$ & 0.116 \\
\hline Adjusted & 1.071 & $1.048,1.095$ & 1.092 & $1.068,1.115$ & 1.087 & $1.063,1.111$ & 1.087 & $1.059,1.115$ & $0 \cdot 300$ \\
\hline
\end{tabular}

Covariates included in the adjusted mixed model include smoking, intervention group, vitamin D and Ca supplementation, disease or medication reducing BMD, age, height, weight, duration of hormone therapy and energy intake.

( $\mathrm{g} / \mathrm{d}, \%$ of energy) and fibre, and with the lowest fat intake $(\mathrm{g} / \mathrm{d}$, \% of energy). Participants in the highest BSD score quartile had also the greatest intakes of $\mathrm{Ca}$, vitamin $\mathrm{D}$ and vitamin $\mathrm{C}$.

Higher MD scores were associated with higher consumption of fruit and berries as well as vegetables (Table 2). On the contrary, fish intake was higher in the lowest MD category as compared with the highest category. Energy intake was higher in the highest quartile than in the lowest and second quartiles. Carbohydrate and fibre intakes as well as PUFA:SFA were higher in the higher MD quartiles.

Quartiles of BSD and MD scores were not associated with femoral neck, lumbar or total body BMD in unadjusted or adjusted models (Table 3). Neither were there significant associations in the control group only (data not shown). In addition to quartiles, we carried out the statistical analysis using BSD and MD scores in tertile categories, and the results showed no significant differences in total, femoral and lumbar BMD in the BSD or MD tertiles (data not shown).

Most of the adjusted associations of BSD and MD score components with BMD were not significant, with the exceptions of fat quality as well as alcohol and fruit consumption. Higher MUFA + PUFA:SFA ratio was significantly associated with lumbar BMD (1.090 (95\% CI $1.045,1.135)$ and 1.131 (95\% CI $1.098,1.165) \mathrm{g} / \mathrm{cm}^{2}$ in categories of lower and greater than or equal to the median ratio, respectively, $P=0.035)$ and femur BMD (0.856 (95\% CI $0.827,0.885)$ and 0.860 (95\% CI 0.826 , $0.894) \mathrm{g} / \mathrm{cm}^{2}$ in categories of lower and greater than or equal to the median ratio, respectively, $P=0 \cdot 037$ ).
Alcohol consumption exceeding 1 portion/week was associated with higher BMD at lumbar (1.167 (95\% CI $1.128,1.207)$ and $1.088(95 \%$ CI $1.054,1.123) \mathrm{g} / \mathrm{cm}^{2}$, respectively, $P<0.001$ ), femoral neck (0.894 (95\% CI 0.867, $0.920)$ and $0.861(95 \%$ CI $0.839,0.884) \mathrm{g} / \mathrm{cm}^{2}$, respectively, $P=0.013)$ and total body (1.097 (95\% CI 1.076, 1.117) and $1.063(95 \%$ CI $1.044,1.081) \mathrm{g} / \mathrm{cm}^{2}$, respectively, $P=0.001$ ) sites compared with lower consumption. Fruit consumption quartile from the BSD score was associated with total body BMD (quartile 1: 1.069 (95\% CI 1.048, 1.091) g/ $\mathrm{cm}^{2}$; quartile 2: 1.095 (95\% CI 1.073, 1.118) g/ $\mathrm{cm}^{2}$; quartile 3: 1.065 (95\% CI $1.043,1.086) \mathrm{g} / \mathrm{cm}^{2}$; quartile $4: 1.082$ (95\% CI 1.060 , 1.104) $\left.\mathrm{g} / \mathrm{cm}^{2} ; P=0 \cdot 046\right)$.

\section{Discussion}

The present study assessed the relationship of two dietary patterns, BSD and MD, with BMD in elderly women. Neither dietary pattern was significantly associated with BMD. The dietary patterns did, however, capture overall healthy characteristics of dietary intake.

BSD has earlier been associated with other health outcomes but associations with bone health have not been reported. Higher adherence to the BSD was associated with lower risk of abdominal obesity and lower C-reactive protein as a marker for inflammation ${ }^{(17,19)}$ and furthermore with better physical function ${ }^{(20)}$. On the contrary, the BSD was not related to type 2 diabetes risk ${ }^{(29)}$ and was related to decreased HDL cholesterol ${ }^{(17)}$.

Regarding the MD and BMD, the results have been controversial $^{(30)}$. Adherence to the MD was not associated 
with BMD in a cross-sectional setting in 220 Greek adult women $^{(10)}$ or in 1180 Portuguese adolescents ${ }^{(31)}$; on the contrary, a significant association was reported in 200 Spanish women ${ }^{(12)}$. MD supplemented with nuts or virgin olive oil did not affect BMD in a 1-year intervention ${ }^{(32)}$, which might have been too short a time to observe an effect. Even though MD adherence as such has yielded mixed results in relation to BMD, components of the MD like fruit, vegetables, fish, olive oil and low red meat consumption have been associated with $\mathrm{BMD}^{(10,12)}$. We did not observe such associations, except a weak association for fruit intake. Inconsistent results could result from differences in study populations, small subject numbers and it is of note that most of the earlier studies were cross-sectional.

Use of predefined dietary patterns allows easy comparison of results with dietary recommendations and other studies, as well as interpretation to practical dietary recommendations. In addition to the BSD and MD, there are other predefined dietary patterns that have been studied in relation to bone health. Better-quality diet as indicated by higher Alternative Healthy Eating Index (AHEI) was associated with lower risk of hip fracture in Singapore Chinese aged 45-74 years ${ }^{(33)}$. However, AHEI was not associated with peak bone mass in women aged 18-30 years ${ }^{(13)}$, nor was HEI-2005 associated with bone turnover markers in women aged $>45$ years $^{(34)}$. The Dietary Approaches to Stop Hypertension (DASH) diet was not related to BMD in adolescents ${ }^{(31)}$, even though that it is characterized by high intakes of fruit and vegetables and low-fat dairy products. A simpler Oslo Health Study dietary index based on the ratio of consumption of soft drinks to that of fruit, berries, fruit juices and vegetables was negatively associated with BMD in adults ${ }^{(35)}$, but not in adolescents ${ }^{(31)}$.

The predefined dietary patterns have thus shown inconsistent associations with BMD and there were no significant associations in our study. Even so, we would argue that the BSD and MD were able to capture healthy dietary characteristics. Better adherence to the BSD was associated with higher fruit and berries, vegetable, fish and low-fat dairy product consumption and better adherence to the MD was associated with higher fruit and berries and vegetable consumption. Both higher BSD and MD scores were associated with better dietary fat quality as shown by PUFA:SFA. The range of BSD score in our study was comparable to that reported earlier in three Finnish data sets ${ }^{(17)}$, which would indicate that the variability in the score was reliable. Even though the BSD classifies individuals according to overall dietary quality and it relates to other health outcomes, it may not capture adequately the dietary factors that are most relevant for bone health.

Of the components of BSD and MD scores, only MUFA +PUFA:SFA and alcohol and fruit consumption were associated with BMD. This is in line with our earlier results showing that PUFA intake was positively associated with
BMD at the lumbar spine and total body ${ }^{(22)}$ and that low to moderate alcohol intake was positively associated with BMD at the lumber spine and femoral neck ${ }^{(36)}$. It has been suggested that light to moderate alcohol intake may decelerate the rate of bone remodelling in older individuals ${ }^{(37)}$. Fruit and vegetables have been associated with better bone health at multiple measurement sites ${ }^{(14,38,39)}$. Other components of the scores were not associated with BMD in the current data, which contrasts with several earlier studies. Dairy products have been found to be beneficial $^{(9,11,14)}$. Greater amount of oily fish consumption has been indicated to have a positive influence on $\mathrm{BMD}^{(10,11,40)}$; whereas greater intake of protein from red meat and processed food had a negative relationship with $\operatorname{BMD}^{(9,11,14)}$. The results on associations of diet with different bone sites (trabecular or cortical) have been mixed $^{(8,41)}$.

Our study has strengths and limitations. BMD was measured with dual-energy X-ray absorptiometry, which is a reliable determinant for bone health; and low BMD is strongly associated with osteoporotic fractures ${ }^{(42)}$. The duration of 3 years was selected for the original intervention and is regarded as long enough ${ }^{(43)}$, and it is also quite comparable to the cohort studies with 1-6 years of follow-up ${ }^{(8,32,44)}$. BMD in the present OSTPRE-FPS did not differ from the whole OSTPRE study ${ }^{(21)}$, which is a population-based sample. Population-based design with random selection of the sub-sample is a strength of the present study, even though it was weakened by a response rate of $63.5 \%$. For dietary assessment, we used the data from $3 \mathrm{~d}$ food records, which are regarded as the gold standard or reference method for dietary assessment and are used to validate other nutritional assessment methods ${ }^{(45)}$. The $3 \mathrm{~d}$ food records, however, have limitations in assessment of habitual long-term diet and they do not capture infrequently consumed foods such as fish, which is typically consumed on 1-2 d/week. We did not have information on possible changes in food consumption during the 3-year period due to one baseline assessment. However, all dietary assessment tools include bias sources, and it is suggested that food records could be more accurate than FFQ in assessing absolute intakes when compared with biomarkers even though that is not directly applicable to long-term dietary intake which is relevant in relation to changes in bone health ${ }^{(46,47)}$. In addition, since the dietary assessment was conducted using self-reported data and accordingly relied on participants' accuracy, reporting errors are possible. The non-significant results may be related to the number of participants that limited the power of the analyses. Similarly, due to small number of Finnish elderly women, caution should be taken in generalization of the results to other elderly populations. Although we adjusted for several recognized confounding factors ${ }^{(5,42)}$, we cannot exclude the possibility that the results were affected by factors that we were not able to control. Regarding BMD, 
the interactions of food consumption, body composition and energy intake and their changes can be difficult to control $^{(7,48)}$.

\section{Conclusion}

Neither of the dietary scores, the BSD or MD, was significantly associated with BMD in elderly women. The results suggest that these dietary patterns may not adequately reflect dietary factors relevant to bone health.

\section{Acknowledgements}

Financial support: The OSTPRE-FPS was supported by the North Savo Regional Fund of the Finnish Cultural Foundation (Hulda Tossavainen Foundation), Sigrid Juselius Foundation, Academy of Finland and Kuopio University Hospital EVO grant. Funders had no role in the design, analysis or writing of this article. Conflict of interest: None. Authorship: The original study was planned by M.T. and H.K. This research question was planned by A.T.E. and H.S. The statistical analyses were carried out by A.T.E., H.S. and M.I. The first draft was written by H.S. and A.T.E., and all the authors contributed to critically revising it. Ethics of buman subject participation: The study was approved in October 2001 by the ethical committee of Kuopio University Hospital, and the subjects were involved voluntarily. Written informed consent was obtained from all subjects. The study was registered in Clinical trials.gov by the identification NCT00592917.

\section{References}

1. United Nations Population Fund (2012) Ageing in the twenty-first century: a celebration and a challenge. http:// www.unfpa.org/sites/default/files/pub-pdf/Ageing\%20report. pdf (accessed October 2016).

2. Briggs AM, Cross MJ, Hoy DG et al. (2016) Musculoskeletal health conditions represent a global threat to healthy aging: a report for the 2015 World Health Organization World Report on Ageing and Health. Gerontologist 56, Suppl. 2, S243-S255.

3. Nguyen ND, Pongchaiyakul C, Center JR et al. (2005) Identification of high-risk individuals for hip fracture: a 14-year prospective study. J Bone Miner Res 20, 1921-1928.

4. Korhonen N, Niemi S, Parkkari J et al. (2013) Continuous decline in incidence of hip fracture: nationwide statistics from Finland between 1970 and 2010. Osteoporos Int 24, 1599-1603.

5. Curtis E, Litwic A, Cooper C et al. (2015) Determinants of muscle and bone aging. J Cell Physiol 230, 2618-2625.

6. Dawson-Hughes B \& Harris S (2002) Calcium intake influences the association of protein intake with rates of bone loss in elderly men and women. Am J Clin Nutr 75, $773-779$.

7. Isanejad M, Sirola J, Mursu J et al. (2017) Association of protein intake with bone mineral density and bone mineral content among elderly women: the OSTPRE Fracture Prevention Study. J Nutr Health Aging 21, 622-630.
8. Pedone C, Napoli N, Pozzilli P et al. (2011) Dietary pattern and bone density changes in elderly women: a longitudinal study. J Am Coll Nutr 30, 149-154.

9. Mangano KM, Sahni S, Kiel DP et al. (2015) Bone mineral density and protein-derived food clusters from the Framingham Offspring Study. J Acad Nutr Diet 115 , 1605-1613.

10. Kontogianni MD, Melistas L, Yannakoulia M et al. (2009) Association between dietary patterns and indices of bone mass in a sample of Mediterranean women. Nutrition 25, $165-171$.

11. Denova-Gutiérrez E, Clark P, Tucker KL et al. (2016) Dietary patterns are associated with bone mineral density in an urban Mexican adult population. Osteoporos Int 27, 3033-3040.

12. Rivas A, Romero A, Mariscal-Arcas M et al. (2013) Mediterranean diet and bone mineral density in two age groups of women. Int J Food Sci Nutr 64, 155-161.

13. Zagarins SE, Ronnenberg AG, Gehlbach SH et al. (2012) Are existing measures of overall diet quality associated with peak bone mass in young premenopausal women? J Hum Nutr Diet 25, 172-179.

14. Shin S, Sung J \& Joung H (2015) A fruit, milk and whole grain dietary pattern is positively associated with bone mineral density in Korean healthy adults. Eur J Clin Nutr 69, 442-448.

15. de Franca NAG, Camargo MBR, Lazaretti-Castro $M$ et al. (2016) Dietary patterns and bone mineral density in Brazilian postmenopausal women with osteoporosis: a cross-sectional study. Eur J Clin Nutr 70, 85-90.

16. Kanerva N, Kaartinen NE, Schwab U et al. (2014) The Baltic Sea Diet Score: a tool for assessing healthy eating in Nordic countries. Public Health Nutr 17, 1697-1705.

17. Kanerva N, Kaartinen NE, Rissanen H et al. (2014) Associations of the Baltic Sea diet with cardiometabolic risk factors - a meta-analysis of three Finnish studies. Br J Nutr 112, 616-626.

18. Kanerva N, Kaartinen NE, Schwab U et al. (2013) Adherence to the Baltic Sea diet consumed in the Nordic countries is associated with lower abdominal obesity. Br J Nutr 109, $520-528$

19. Kanerva N, Loo B, Eriksson JG et al. (2014) Associations of the Baltic Sea diet with obesity-related markers of inflammation. Ann Med 46, 90-96.

20. Perälä M, von Bonsdorff M, Männistö S et al. (2016) A healthy Nordic diet and physical performance in old age: findings from the longitudinal Helsinki Birth Cohort Study. Br J Nutr 115, 878-886.

21. Kärkkäinen M, Tuppurainen M, Salovaara K et al. (2010) Effect of calcium and vitamin D supplementation on bone mineral density in women aged 65-71 years: a 3-year randomized population-based trial (OSTPRE-FPS). Osteoporos Int 21, 2047-2055.

22. Järvinen R, Tuppurainen M, Erkkilä AT et al. (2012) Associations of dietary polyunsaturated fatty acids with bone mineral density in elderly women. Eur J Clin Nutr 66, 496-503.

23. Isanejad M, Sirola J, Mursu J et al. (2017) Association of the Baltic Sea and Mediterranean diets with indices of sarcopenia in elderly women, OSPTRE-FPS study. Eur J Nutr (Epublication ahead of print version).

24. Eloranta AM, Schwab U, Venäläinen T et al. (2016) Dietary quality indices in relation to cardiometabolic risk among Finnish children aged 6-8 years - the PANIC study. Nutr Metab Cardiovasc Dis 26, 833-841.

25. Tognon G, Rothenberg E, Eiben G et al. (2011) Does the Mediterranean diet predict longevity in the elderly? A Swedish perspective. Age 33, 439-450.

26. Sofi F, Macchi C, Abbate R et al. (2014) Mediterranean diet and health status: an updated meta-analysis and a proposal 
for a literature-based adherence score. Public Health Nutr 17, 2769-2782.

27. Bamia C, Lagiou P, Buckland G et al. (2013) Mediterranean diet and colorectal cancer risk: results from a European cohort. Eur J Epidemiol 28, 317-328.

28. Gueorguieva R \& Krystal JH (2004) Move over ANOVA: progress in analyzing repeated-measures data and its reflection in papers published in the Arch Gen Psychiatry 61, 310-317.

29. Kanerva N, Rissanen H, Knekt P et al. (2014) The healthy Nordic diet and incidence of type 2 diabetes - 10-year follow-up. Diabetes Res Clin Pract 106, E34-E37.

30. Romero Perez A \& Rivas Velasco A (2014) Adherence to Mediterranean diet and bone health. Nutr Hosp 29 , 989-996.

31. Monjardino T, Lucas R, Ramos E et al. (2014) Associations between $a$ priori-defined dietary patterns and longitudinal changes in bone mineral density in adolescents. Public Health Nutr 17, 195-205.

32. Bullo M, Amigo-Correig P, Marquez-Sandoval F et al. (2009) Mediterranean diet and high dietary acid load associated with mixed nuts: effect on bone metabolism in elderly subjects. J Am Geriatr Soc 57, 1789-1798.

33. Dai Z, Butler LM, van Dam RM et al. (2014) Adherence to a vegetable-fruit-soy dietary pattern or the Alternative Healthy Eating Index is associated with lower hip fracture risk among Singapore Chinese. J Nutr 144, 511-518.

34. Hamidi M, Tarasuk V, Corey P et al. (2011) Association between the Healthy Eating Index and bone turnover markers in US postmenopausal women aged $\geq 45 \mathrm{y}$. $A m \mathrm{~J}$ Clin Nutr 94, 199-208.

35. Høstmark AT, Søgaard AJ, Alvær K et al. (2011) The Oslo Health Study: a dietary index estimating frequent intake of soft drinks and rare intake of fruit and vegetables is negatively associated with bone mineral density. $J$ Osteoporos 2011, 102686.

36. Sommer I, Erkkilä AT, Järvinen R et al. (2013) Alcohol consumption and bone mineral density in elderly women. Public Health Nutr 16, 704-712.

37. Gaddini GW, Turner RT, Grant KA et al. (2016) Alcohol: a simple nutrient with complex actions on bone in the adult skeleton. Alcohol Clin Exp Res 40, 657-671.
38. Hardcastle AC, Aucott L, Fraser WD et al. (2011) Dietary patterns, bone resorption and bone mineral density in early post-menopausal Scottish women. Eur J Clin Nutr 65, 378-385.

39. Karamati M, Yousefian-Sanni M, Shariati-Bafghi $\mathrm{S}$ et al. (2014) Major nutrient patterns and bone mineral density among postmenopausal Iranian women. Calcif Tissue Int 94, 648-658.

40. Zalloua P, Hsu Y, Terwedow $\mathrm{H}$ et al. (2007) Impact of seafood and fruit consumption on bone mineral density. Maturitas 56, 1-11.

41. Corwin RL, Hartman TJ, Maczuga SA et al. (2006) Dietary saturated fat intake is inversely associated with bone density in humans: analysis of NHANES III. $J$ Nutr 136, 159-165.

42. Kanis JA, McCloskey EV, Johansson H et al. (2013) European guidance for the diagnosis and management of osteoporosis in postmenopausal women. Osteoporos Int $\mathbf{2 4}$, 23-57.

43. Weaver CM, Gordon CM, Janz KF et al. (2016) The National Osteoporosis Foundation's position statement on peak bone mass development and lifestyle factors: a systematic review and implementation recommendations. Osteoporos Int 27, 1281-1386.

44. Shams-White MM, Chung M, Du M et al. (2017) Dietary protein and bone health: a systematic review and metaanalysis from the National Osteoporosis Foundation. Am J Clin Nutr 105, 1528-1543.

45. Tucker KL (2007) Assessment of usual dietary intake in population studies of gene-diet interaction. Nutr Metab Cardiovasc Dis 17, 74-81.

46. Freedman LS, Potischman N, Kipnis V et al. (2006) A comparison of two dietary instruments for evaluating the fatbreast cancer relationship. Int J Epidemiol 35, 1011-1021.

47. Prentice RL, Mossavar-Rahmani Y, Huang Y et al. (2011) Evaluation and comparison of food records, recalls, and frequencies for energy and protein assessment by using recovery biomarkers. Am J Epidemiol 174, 591-603.

48. Langsetmo L, Poliquin S, Hanley DA et al. (2010) Dietary patterns in Canadian men and women ages 25 and older: relationship to demographics, body mass index, and bone mineral density. BMC Musculoskelet Disord 11, 20. 